\title{
Construcción de conocimiento en alumnos universitarios a partir de la modelización matemática
}

\author{
Construction of knowledge in university students from mathematical modeling
}

\author{
L. Arias-Montoya iD, J. Deulofeu-Piquet iD
}

\begin{abstract}
Resumen - La enseñanza en el aula de clase universitaria es una construcción pedagógica que tiene en cuenta al individuo, su formación y su interacción con el medio. Ese es el planteamiento que se pretende desarrollar y validar a lo largo del presente artículo de investigación, a partir de la interacción con estudiantes de ingeniería industrial de la Universidad Tecnológica de Pereira. El primer acercamiento práctico de la presente investigación se da a partir del encuentro inicial del investigador con los estudiantes de la asignatura ingeniería económica del programa de Ingeniería Industrial (séptimo semestre) de la Universidad Tecnológica de Pereira, espacio que permitió conocer $y$ comprender algunas de las facetas vivenciadas por los estudiantes durante su formación profesional, haciendo de la cotidianidad del aula el escenario propicio para determinar el grado de aprendizaje real de los estudiantes. El estudio es de carácter descriptivo, con una estructura metodológica que responde al estudio de caso, que recurre a la entrevista de estudiantes como el principal instrumento de obtención de información primaria. Para la lectura de los datos se emplea el software ATLAS.ti (versión 8.1), herramienta que facilita la gestión de los conceptos que participan de esta experiencia investigativa, al tiempo que favorece la labor de interpretación de los aprendizajes adquiridos por los estudiantes durante su proceso de formación académica. Posteriormente se aplica una unidad didáctica, diseñada para valorar la práctica educativa en la que participan ${ }^{3}$ el profesor y los estudiantes. De esta experiencia de aula se analizan los factores pedagógicos que median el proceso de enseñanza-aprendizaje, a la luz de los fundamentos conceptuales sobre la construcción de conocimiento y modelización matemática (en forma de narrativa sobre las experiencias construidas centradas en las entrevistas) tomados de diversos
\end{abstract}

Este manuscrito fue enviado el 14 de noviembre de 2018 y aceptado el 25 de junio de 2019.

Es un artículo de investigación científica derivado de la tesis doctoral "Construcción de conocimiento en alumnos universitarios a partir de la modelización matemática" del Doctorado en Didáctica de la Universidad Tecnológica de Pereira.

L. Arias-Montoya, profesor titular del programa Ingeniería Industrial, Universidad Tecnológica de Pereira, Colombia, Calle 27\#10-02. (leoarias@utp.edu.co).

J. Deulofeu-Piquet, profesor titular del Departamento de Didáctica de las Matemáticas y de las Ciencias, Universidad Autónoma de Barcelona, España, Facultad de Educación. Edificio G5, despacho 132. Cerdanyola del Vallés, 08193. (jordi.deulofeu@uab.cat). autores y de la ingeniería industrial. Con la creación y puesta en marcha de la unidad didáctica al igual que con la ayuda de la fundamentación conceptual, surge, se desarrolla e implementa esta propuesta investigativa, a través de la comprensión y percepción del individuo dentro de su entorno.

Palabras clave - construcción de conocimiento, modelización matemática, zona de desarrollo próximo, zona de desarrollo real.

Abstract - The university classroom teaching is a pedagogic construction that considers the subject, his training and his environment interaction. This approach is going to be developed and validated in this paper. The first practical approach to research is an encounter of the researcher with Economic Engineering students, a subject oriented in seven semester of Industrial Engineering at UTP. This encounter allows knowing and understanding some facets that students live in their professional training, which makes the classroom everydayness the propitiated stage to establish the real student's learning level. This investigation is a descriptive research focused on a methodological structure based in the case study, which resorts to the students' interview as the main instrument for obtaining primary information. The software used to read the data is ATLAS.ti V.8.1, which facilitates the concepts' management in this investigative experience at the same time that favors an interpretation of the learning acquired by students during their academic training process. In the investigation process, a didactic unit is applied and designed to value teachers and students participation in educational practice. From this classroom experience, the author analyzes the pedagogical factors that mediate the teaching-learning process, considering conceptual foundations on the knowledge construction and mathematical modeling (in a narrative form about built experiences centered on interviews), taking diverse authors' ideologies and industrial engineering experiences. This research proposal is arise, developed and implemented by the creation and implementation of the didactic unit, and conceptual foundation use, through an understanding and perception of the individual within its environment.

Index Terms - knowledge construction, mathematical modeling, proximal development zone, and real development zone. 


\section{INTRODUCCIÓN}

$\mathrm{L}$ A investigación que se despliega en el presente artículo pretende evidenciar la relación que existe entre la construcción de conocimiento y la modelización matemática, a partir de la aplicación de una unidad didáctica a estudiantes universitarios de ingeniería industrial de séptimo semestre, en temas de evaluación financiera de proyectos, particularmente, la tasa interna de retorno (TIR).

La construcción de conocimiento ha sido un tema ampliamente abordado desde diferentes posturas. Partiendo de perspectivas como el cognitivismo y el constructivismo, cada una presenta planteamientos diferentes y particulares que limitan la noción de aprendizaje.

Este artículo comprende conceptos relativos a la construcción de conocimiento, a partir de enfoques cognitivistas y constructivistas relacionados con el aprendizaje, el papel de la memoria y la transferencia de conocimiento. Igualmente, comprende conceptos de modelización matemática, pero con un interés central: evidenciar la incidencia de la modelización matemática en la construcción de conocimiento.

La construcción de conocimiento se aborda desde los planteamientos de teóricos como Piaget, documentado por Parrat [1]; de Bruner, documentado por Guilar [2]; de Vygotsky [3], y de Ertmer y Newby [4], quienes son los principales referentes sobre este tema. Se parte de la idea de que las teorías constructivistas y cognitivistas se complementan al integrar el proceso cognitivo del estudiante con sus experiencias, incorporación que es parte integral de la construcción del aprendizaje.

De manera complementaria, se aborda la hipótesis de que la modelización matemática es una metodología que facilita la construcción de conocimiento en el estudiante. En tal sentido, se parte de las investigaciones de Maaß [5] y Kaiser y Schwarz [6], quienes abordan el tema de la modelización matemática y las competencias requeridas para modelizar la realidad. Estos antecedentes presentan aportes muy valiosos, toda vez que consideran temas altamente relevantes al respecto de las competencias que debe desarrollar el estudiante cuando desea representar la realidad y modelar su comportamiento. Estas competencias complementan aquellas que con relación a la construcción de conocimiento se abordan desde los autores, y abren la posibilidad de integrar un nuevo enfoque de construcción de aprendizaje mediante la inclusión de factores de modelización.

A partir de los resultados de esta experiencia investigativa se construye una propuesta de enseñanza-aprendizaje enfocada en la construcción de conocimiento mediante la modelización matemática, el aprendizaje cooperativo y el aprendizaje significativo, en una convergencia de planteamientos en la que el trabajo en equipo, el metaaprendizaje, el razonamiento crítico y la creación de modelos representativos de la realidad permiten la ubicación del estudiante en la zona de desarrollo real, que es aquella en la que se considera que ha logrado la apropiación de conocimiento, en una integración constructiva de pensar, hacer y sentir [7]. Desde allí, lo que se busca es transformar al estudiante en un sujeto activo en el proceso de construcción de conocimiento, dispuesto a un aprendizaje por descubrimiento, y abierto a la apropiación de significados.

\section{METODOLOGÍA}

El método utilizado es el estudio de caso. La experiencia se desarrolló con un grupo de estudiantes de la asignatura de ingeniería económica del programa de Ingeniería Industrial de la Universidad Tecnológica de Pereira. En este caso, la terna estudiantes-profesor- actividad matemática se toma como un todo. El grupo de trabajo estuvo conformado por 32 estudiantes, de los que se seleccionó una muestra de 18 . La muestra se dividió en tres grupos de 4 estudiantes y dos grupos de 3 estudiantes, utilizando la técnica de los mini grupos focales. Una de las razones para seleccionar a estos estudiantes es que cuentan con conocimientos o experiencias especializadas para discutir en grupo [8].

Es necesario que los estudiantes que participan en la unidad didáctica cuenten con un amplio bagaje conceptual, ya que esos conocimientos son el pilar que soporta la construcción de nuevos significados y de los conceptos aplicados en la asignatura. En este caso, los cinco grupos se reúnen 4 veces. A través de la resolución de una serie de ejercicios, se busca observar el grado de construcción grupal. Asimismo, también se plantean una serie de ejercicios individuales, pero con el propósito de evidenciar el grado de construcción individual.

Como el interés del autor es determinar la incidencia de la modelización matemática en la construcción de conocimiento en los estudiantes, se consideró que la entrevista es el mejor instrumento para recolectar información concerniente a su percepción de la metodología. Para ello, se comparó el grado de aceptación de la metodología tradicional frente a la metodología aplicada en la unidad didáctica.

Los instrumentos para la gestión de la información son los programas Excel (Windows) y ATLAS.ti (versión 8.1). Como se trata de herramientas que facilitan el análisis cuantitativo y cualitativo de la información, apoyan el proceso de determinar si existen relaciones entre los resultados obtenidos y las propuestas teóricas de los autores consultados, con el propósito de dar respuesta a la hipótesis planteada por el autor.

\section{RESULTADOS}

\section{A. Referentes teóricos}

La investigación se documenta con escritos que versan sobre la construcción de conocimiento. Los autores base son Vygotsky [3], Piaget [1], Bruner [2], Ertmer y Newby [4], las revisiones de modelización matemática de Maaß [5], y Kaiser y Schwartz [6]. Se trata de autores que desde sus textos hacen importantes aportes para la construcción de conocimiento.

Uno de los aportes más representativos es el de Vygotsky [3], quien hace referencia a la zona de desarrollo próximo. Es un aspecto que resulta fundamental para la investigación 
debido a su concepción misma, pues se entiende como la distancia que toma alcanzar la zona de desarrollo real.

La zona de desarrollo real es aquella en la que el estudiante presenta un nivel de apropiación de conocimiento, y puede resolver problemas sin la mediación de un compañero o facilitador. Esta zona se caracteriza en la investigación como una combinación entre la apropiación, el aprendizaje por descubrimiento, la categorización y la zona de desarrollo real, e implica que el estudiante construye conocimiento, y tiene la capacidad para aplicarlo en diferentes contextos. Estos últimos fueron expuestos y evidenciados a partir de los modelos matemáticos que se sustentan como representación de la realidad.

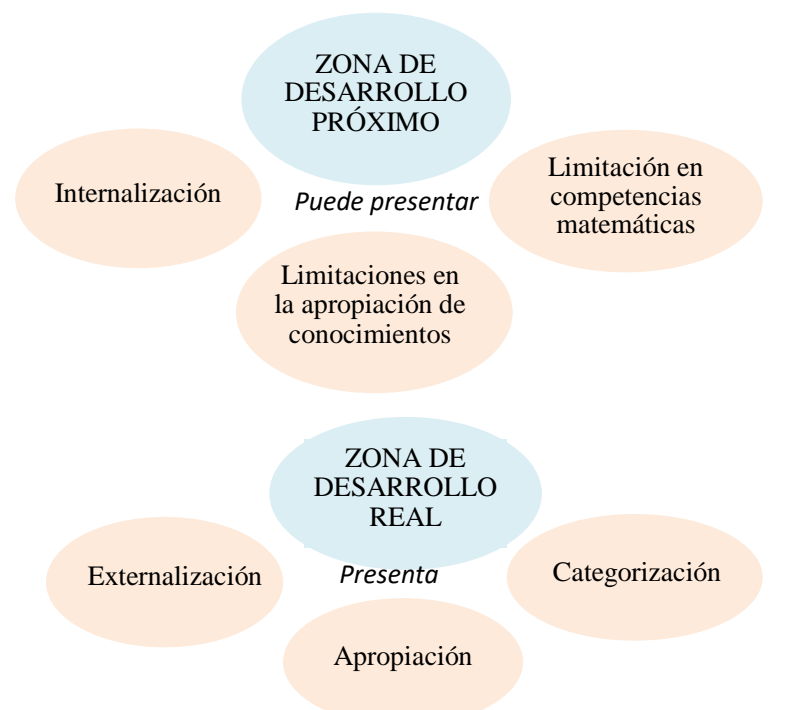

Fig. 1. Zonas de desarrollo planteadas por Vygotsky.

La capacidad de modelamiento matemático se relaciona con la construcción real de conocimiento cuando el estudiante presenta una apropiación de los conceptos -exponiendo satisfactoriamente los modelos matemáticos que lo soportany, simultáneamente, despliega capacidades para expresar verbalmente sus ideas sobre los conceptos expuestos en clase. Así, trascendiendo de la internalización a la externalización, demuestra una total construcción de conocimiento.

De la literatura consultada se extraen una serie de códigos de análisis, que son la base de la investigación.

TABLA I

DEFINICIÓN DE CóDIGOS DE ANÁLISIS

\begin{tabular}{ll}
\hline \hline & Códigos de análisis \\
\hline \multirow{2}{*}{$\begin{array}{l}\text { Representación de la } \\
\text { realidad }\end{array}$} & Enactivo: a partir de reacciones \\
& $\begin{array}{l}\text { Icónico: a partir de imágenes } \\
\text { Simbólico: a partir de símbolos }\end{array}$ \\
Relación situacional & $\begin{array}{l}\text { Relación entre conceptos y experiencias } \\
\text { Contextos significativos }\end{array}$ \\
& Permanencia entre la realidad y las matemáticas \\
Modelización & Uso del conocimiento y monitoreo del proceso \\
matemática & Entendimiento y simplificación del mundo real \\
& Escogencia de las variables claves de intervención \\
\hline
\end{tabular}

\begin{tabular}{|c|c|}
\hline \multirow{2}{*}{ Meta-aprendizaje } & $\begin{array}{l}\text { Conocimiento propio a partir de su propio } \\
\text { pensamiento }\end{array}$ \\
\hline & Control de los procesos cognitivos previos \\
\hline \multirow{4}{*}{ Internalización } & Codificación interna de la información \\
\hline & Actividades mentales que conducen a una respuesta \\
\hline & $\begin{array}{l}\text { Almacenamiento organizado y significativo de la } \\
\text { información }\end{array}$ \\
\hline & Reconstrucción interna \\
\hline Externalización & Competencia para la transmisión clara de ideas \\
\hline \multirow{2}{*}{$\begin{array}{l}\text { Aprendizaje por } \\
\text { descubrimiento }\end{array}$} & Aprendizaje por invención \\
\hline & $\begin{array}{l}\text { Descubre relaciones entre conceptos y construye } \\
\text { conocimiento }\end{array}$ \\
\hline \multirow{3}{*}{ Apropiación } & Nuevo escalón de enseñanza-aprendizaje \\
\hline & $\begin{array}{l}\text { Adquisición de una nueva herramienta cultural } \\
\text { (lectura, escritura, notación matemática) }\end{array}$ \\
\hline & Formación de conceptos \\
\hline \multirow{2}{*}{ Categorización } & Toma de decisiones \\
\hline & $\begin{array}{l}\text { Clasificación de los datos del exterior a través de } \\
\text { las categorías de las que dispone para entenderlo } \\
\text { Comprender el mundo real }\end{array}$ \\
\hline \multirow{4}{*}{$\begin{array}{l}\text { Competencias } \\
\text { matemáticas }\end{array}$} & Resolver matemáticamente preguntas \\
\hline & Interpretar resultados matemáticos \\
\hline & Validar la solución \\
\hline & Reconocer variables de influencia \\
\hline \multirow[t]{2}{*}{$\begin{array}{l}\text { Construcción de } \\
\text { relaciones }\end{array}$} & $\begin{array}{l}\text { Afirmaciones del problema para simplificarlo } \\
\text { Relaciones matemáticas con contextos de la } \\
\text { realidad }\end{array}$ \\
\hline & Relaciones entre variables \\
\hline \multirow{5}{*}{$\begin{array}{l}\text { Creación de } \\
\text { significados }\end{array}$} & Entendimiento de la realidad \\
\hline & Interacción con la realidad \\
\hline & Intercambio entre el sujeto y el medio \\
\hline & Construcción a partir de experiencias \\
\hline & Tareas auténticas atadas a contextos significativos \\
\hline \multirow{4}{*}{$\begin{array}{l}\text { Estructura cognitiva } \\
\text { previa }\end{array}$} & $\begin{array}{l}\text { Construcción de conocimiento a partir de } \\
\text { adquisiciones anteriores }\end{array}$ \\
\hline & Trascendencia de la información dada \\
\hline & Apertura de significado y organización \\
\hline & Experiencias y conocimientos previos \\
\hline $\begin{array}{l}\text { Zona de desarrollo } \\
\text { próximo }\end{array}$ & Necesidad de ayuda para resolver un problema \\
\hline $\begin{array}{l}\text { Zona de desarrollo } \\
\text { real }\end{array}$ & Solución independiente de un problema \\
\hline
\end{tabular}

\section{B. Resultados obtenidos}

La unidad didáctica involucra una serie de evaluaciones que se clasifican en función de las codificaciones (o códigos de análisis) localizadas en las propuestas de los autores base (ver Tabla I). El objetivo es determinar si la capacidad de construcción de conocimiento realmente se transforma con la implementación de la estrategia de modelización matemática. Para ello, resulta necesario comparar el grado de construcción de conocimiento con el que los participantes ingresan a la asignatura frente al grado con el que salen luego de aplicar la unidad didáctica.

La primera intervención corresponde a una evaluación de conceptos previos, con la que se pretende determinar el grado de construcción de conocimiento y las zonas de desarrollo en las que los estudiantes se ubican. En la Tabla II se aprecia la forma como los estudiantes respondieron a 6 preguntas del cuestionario. El ejercicio evidenció tres escenarios: a) respuestas correctas (resueltas con los argumentos adecuados); b) respuestas incorrectas (revelan problemas para el buen desempeño); c) preguntas sin respuesta.

El $39 \%$ de los participantes (7 estudiantes) respondió a todas las preguntas, dato que proviene de la sumatoria de su 
ubicación en las zonas de desarrollo. La tabla también dejar ver cuántas preguntas respondieron correctamente y cuántas incorrectamente. Este último caso supone que aún requieren el acompañamiento del profesor.

\begin{tabular}{|c|c|c|c|c|c|c|c|c|c|}
\hline Códigos & 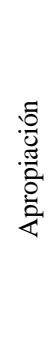 & 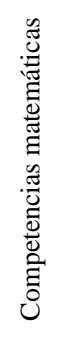 & 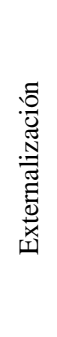 & 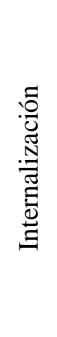 & 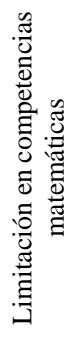 & 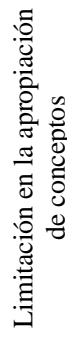 & 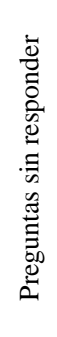 & 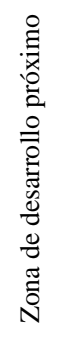 & 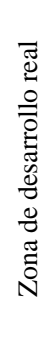 \\
\hline Daniel Andrés & 3 & 1 & 2 & 3 & 0 & 1 & 0 & 4 & 2 \\
\hline Juan David & 1 & 1 & 3 & 0 & 2 & 2 & 1 & 2 & 3 \\
\hline Alejandro & 1 & 1 & 3 & 0 & 2 & 2 & 1 & 2 & 3 \\
\hline Laura Melissa & 0 & 0 & 0 & 1 & 3 & 4 & 1 & 5 & 0 \\
\hline Jennifer & 1 & 1 & 2 & 0 & 2 & 3 & 1 & 3 & 2 \\
\hline Valeria & 0 & 0 & 0 & 0 & 2 & 5 & 0 & 6 & 0 \\
\hline Juan Sebastián & 1 & 1 & 2 & 1 & 2 & 2 & 0 & 3 & 3 \\
\hline Angie Paola & 0 & 0 & 1 & 2 & 2 & 2 & 1 & 4 & 1 \\
\hline Mónica Marcela & 0 & 0 & 1 & 1 & 3 & 3 & 1 & 4 & 1 \\
\hline Victoria & 1 & 2 & 3 & 0 & 1 & 1 & 1 & 0 & 5 \\
\hline Juan José & 3 & 0 & 4 & 0 & 2 & 3 & 0 & 3 & 3 \\
\hline Miguel Ángel & 1 & 0 & 3 & 0 & 2 & 2 & 1 & 3 & 2 \\
\hline Nazly Camila & 1 & 0 & 3 & 0 & 2 & 2 & 1 & 2 & 3 \\
\hline Jhoan Sebastián & 2 & 2 & 2 & 0 & 1 & 1 & 1 & 3 & 2 \\
\hline Geraldine & 0 & 1 & 1 & 0 & 1 & 2 & 0 & 4 & 2 \\
\hline Laura & 2 & 1 & 2 & 0 & 2 & 3 & 1 & 3 & 2 \\
\hline Sara & 1 & 1 & 1 & 0 & 2 & 4 & 0 & 4 & 2 \\
\hline María José & 1 & 1 & 1 & 0 & 1 & 2 & 1 & 3 & 2 \\
\hline
\end{tabular}

TABLA II

Resultados De La Prueba Inicial

Victoria fue la estudiante con el más alto desempeño, en ella 5 de sus respuestas se ubicaron en la zona de desarrollo real. El caso contrario lo representa Valeria, toda vez que sus 6 respuestas estuvieron en la zona de desarrollo próximo. En general, los participantes se ubicaron mayoritariamente en la zona de desarrollo próximo.

Se evidencia que la relación entre los códigos de la Tabla III es cierta cuando los estudiantes se encuentran en una zona de desarrollo real, que es el momento cuando han apropiado el conocimiento, y demuestran competencias matemáticas que les permiten modelar y externalizar sus conocimientos, lo que indica la dependencia de un código y otro.

La zona de desarrollo próximo se puede presentar cuando el estudiante se encuentra en el proceso de internalización, es decir, cuando la diferencia con la zona de desarrollo real es pequeña, y solo se requiere la completa apropiación del tema para poder externalizar la información y alcanzar el nivel real de desarrollo.

A su vez, la zona de desarrollo próximo se puede presentar cuando se involucran variables que limitan la apropiación de un concepto que dificulta la demostración de las competencias matemáticas de los estudiantes. Ello supone la comisión de errores en los cálculos o la errática concepción del problema, haciendo que la diferencia con la zona de desarrollo real sea mayor.

TABLA III RESULTADOS DEL PROCESO

\begin{tabular}{|c|c|c|c|c|}
\hline Código & 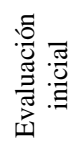 & 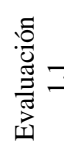 & 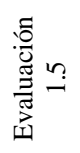 & 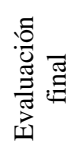 \\
\hline Aprendizaje por descubrimiento & 10 & 0 & 6 & 0 \\
\hline Apropiación & 19 & 15 & 6 & 105 \\
\hline Categorización & 20 & 15 & 18 & 105 \\
\hline Competencias matemáticas & 13 & 15 & 18 & 34 \\
\hline Creación de significado & 28 & 0 & 3 & 104 \\
\hline Desorientación al detalle & 0 & 0 & 0 & 13 \\
\hline Estructura cognitiva previa & 1 & 16 & 18 & 107 \\
\hline Externalización & 35 & 15 & 3 & 104 \\
\hline Internalización & 9 & 0 & 15 & 2 \\
\hline $\begin{array}{l}\text { Limitación en competencias } \\
\text { matemáticas }\end{array}$ & 32 & 1 & 0 & 2 \\
\hline $\begin{array}{l}\text { Limitación en la apropiación de } \\
\text { conceptos }\end{array}$ & 47 & 2 & 12 & 2 \\
\hline Meta-aprendizaje & 11 & 0 & 4 & 62 \\
\hline Modelación matemática & 10 & 14 & 18 & 35 \\
\hline Redescubrimiento & 1 & 0 & 6 & 103 \\
\hline Relación situacional & 3 & 0 & 1 & 0 \\
\hline Representación simbólica & 36 & 17 & 18 & 33 \\
\hline Simplificación de la realidad & 4 & 15 & 18 & 34 \\
\hline Zona de desarrollo próximo & 59 & 2 & 14 & 3 \\
\hline Zona de desarrollo real & 36 & 15 & 4 & 105 \\
\hline
\end{tabular}

A medida que cada estudiante es evaluado, se van revelando una serie de características. Por ejemplo, en la evaluación inicial se encuentran códigos, como el aprendizaje por descubrimiento, que no se manifiestan en las demás evaluaciones individuales. El fenómeno puede encontrar explicación en que en estas fases los estudiantes podían establecer relaciones o conclusiones con base en las explicaciones del docente, sin que este aún haya expuesto las características particulares que presenta en los ejercicios que evalúa. Es el caso de la evaluación 1.5, en la que los estudiantes debían trabajar con dos instrumentos de evaluación de proyectos: la TIR (tasa interne de retorno) y el VPN (valor presente neto). Si bien son dos herramientas empleadas para evaluar proyectos, resultan complementarias. 
De acuerdo con las características particulares de ciertos proyectos, pueden presentar inconsistencias en la evaluación al momento de compararlas, caso que, de hecho, se presenta en esta evaluación. Por un lado, hay estudiantes que explican apropiadamente cómo eligieron una alternativa de inversión usando alguna herramienta financiera, mientras que hay otros que explican el proceso, mas no pueden justificar adecuadamente su selección. Las dos situaciones permiten inferir la zona de desarrollo en la que se encuentra el estudiante. Con el ánimo de ejemplificar ambos casos, se suministran las conclusiones de dos estudiantes (Fig. 2 y 3 ).

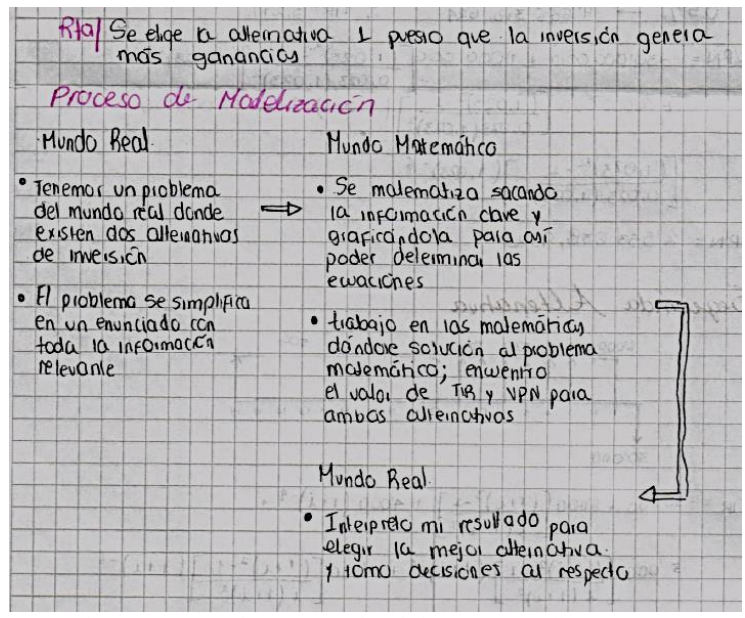

Fig. 2. Conclusiones del ejercicio 1.5 (estudiante Sara).

Sara expone argumentos generales para la escogencia de las alternativas de inversión, pero sin apalancarse en ningún instrumento de evaluación. Es claro que aún se encuentra en el proceso de internalización de las inconsistencias. A pesar de que sus resultados son matemáticamente evidentes, no los menciona en sus conclusiones ni expone un argumento concreto para declarar que la opción uno tiene mayor ganancia. A decir verdad, si se mira desde la TIR, la mayor rentabilidad la tendría la opción dos.

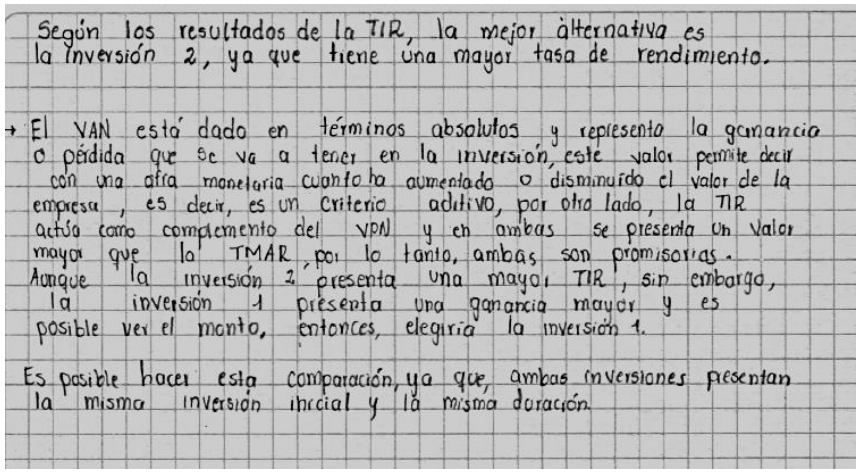

Fig. 3. Conclusiones del ejercicio 1.5 (estudiante Mónica Marcela).

Por su parte, Mónica Marcela expone apropiadamente el porqué de su decisión de invertir en el proyecto 1. Ella realiza una comparación profunda entre las características de cada una de las herramientas de evaluación de proyectos de inversión, no solo desde sus resultados, sino desde su representación. Con ello, valida teóricamente el uso de estas herramientas, denotando además una creación de significado, externalización y metaaprendizaje, aspectos que no evidencia la estudiante anterior.

Por lo tanto, en un ir y venir en la utilización de códigos como el aprendizaje por descubrimiento o la categorización, entre otros (ver viñetas de la imagen), se puede evidenciar la categorización de fases de evaluación. En las Fig. 4, 5 y 6 se asumen esa caracterización.

En la etapa inicial, representada en la evaluación inicial, en los ejercicios 1.5 (resolución individual) y 1.2 (resolución grupal), los estudiantes presentaban una baja estructura cognitiva previa específica en los temas tratados en los talleres. Por lo tanto, se presenta un mayor nivel de aprendizaje por descubrimiento $\mathrm{y}$, en consecuencia, un menor grado de apropiación. El hallazgo encuentra explicación en que los jóvenes están en un proceso de internalización, bajo la premisa de la existencia de limitaciones tanto en el ámbito de competencias matemáticas como en la apropiación de los conceptos. No se presenta un meta-aprendizaje porque los estudiantes están iniciando una aproximación teórica a los conceptos. Igualmente se presenta poco redescubrimiento, y exteriorizan construcción de relaciones y relaciones situacionales, a partir de las cuales pueden crear significados en la mayoría de las situaciones, pero sin fundamentación teórica. Con ello, se concluye que un menor porcentaje de estudiantes está en la zona de desarrollo real, y que la mayoría se encuentra en la zona de desarrollo próximo.

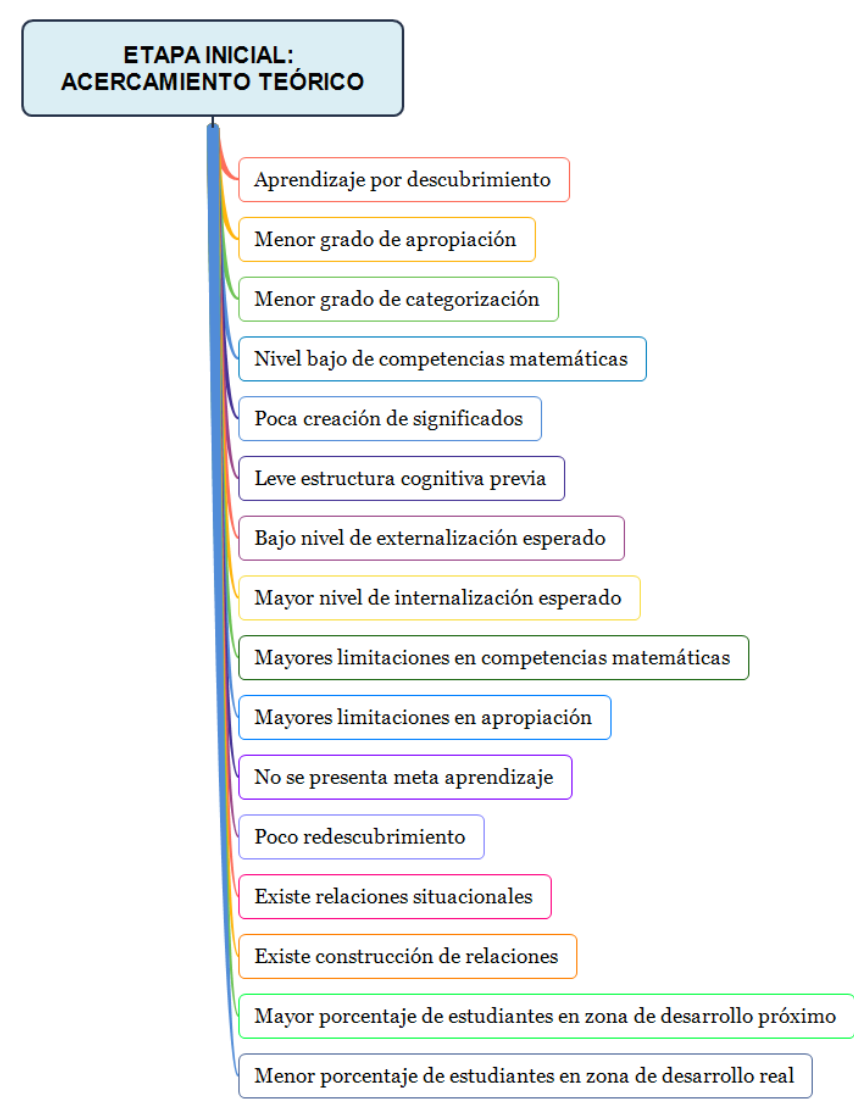

Fig. 4. Caracterización de la etapa inicial de la unidad didáctica [9]. 
Es posible determinar entonces una conexión de códigos en la etapa media de la unidad didáctica, relacionada, en este caso, con los ejercicios 1.1 y 1.4 resueltos individual y grupalmente. De esta forma, en la etapa media es de esperar que los estudiantes todavía presenten cierto nivel de aprendizaje por descubrimiento, dado que no todos los temas han sido suministrados ni tampoco las características más particulares de los ejercicios.

Se presenta un alto nivel de apropiación de conocimiento y de categorización, así como un nivel promedio en las competencias matemáticas, relacionadas, igualmente, con la presencia de limitaciones en competencias matemáticas y en la apropiación.

Dada la connotación de una estructura previa mayor, no se espera la construcción de relaciones como sustento de la construcción de significados. Aún no se ha desarrollado el nivel de externalización requerido, por lo que se está en proceso de redescubrimiento; igualmente, se espera un menor número de personas en zona de desarrollo próximo, para trascender a la zona de desarrollo real.

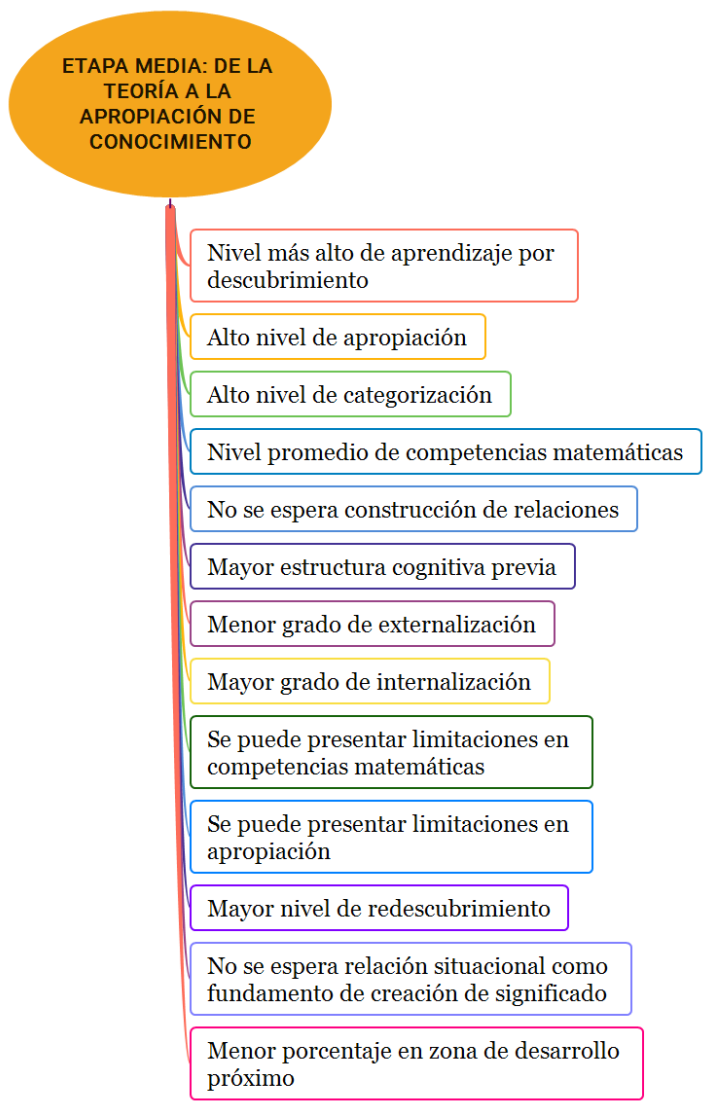

Fig. 5. Caracterización de la etapa media de la unidad didáctica [9]

El diagrama de la etapa final de la unidad didáctica revela, como se esperaba, que se presenta el mayor nivel de apropiación de conocimiento y el menor nivel de aprendizaje por descubrimiento. El resultado confirma que cuando el profesor provee los elementos teórico-prácticos el nivel de categorización y de competencias matemáticas es mayor, lo que implica, a la vez, que no cabe esperar limitaciones en las competencias matemáticas o en la apropiación de conocimiento.

La etapa final de la unidad didáctica presenta los hallazgos encontrados en la investigación a partir de la caracterización de los códigos y la interrelación entre ellos, con el objeto de observar la construcción de conocimiento.

Dado que la etapa final de la unidad didáctica coincide con las fases finales de la asignatura - que es el momento cuando se evalúan los contenidos ya expuestos_-, se esperaba que se presentase un meta-aprendizaje en la exposición de conceptos, hecho que ciertamente ocurrió. Así pues, el instrumento facilitó la creación de las condiciones necesarias para la adecuada generación de los modelos que propician una completa visualización de la realidad, propiciando una transformación real de las fases en las que inicialmente los estudiantes no tenían claros algunos conceptos.

Un ejemplo es la tasa de descuento, concepto sobre el que indagó tanto la evaluación inicial como la final. El balance es que los estudiantes tenían ya tan apropiado el concepto, que fueron capaces de exponerlo claramente en los ejercicios. Esta forma de proceder denota un proceso de transformación lógica que los faculta para construir conceptos autónomamente y ser conscientes de su apropiado uso; asimismo, evidencia que transitaron desde la zona de desarrollo próximo, para ubicarse en la zona de desarrollo real.

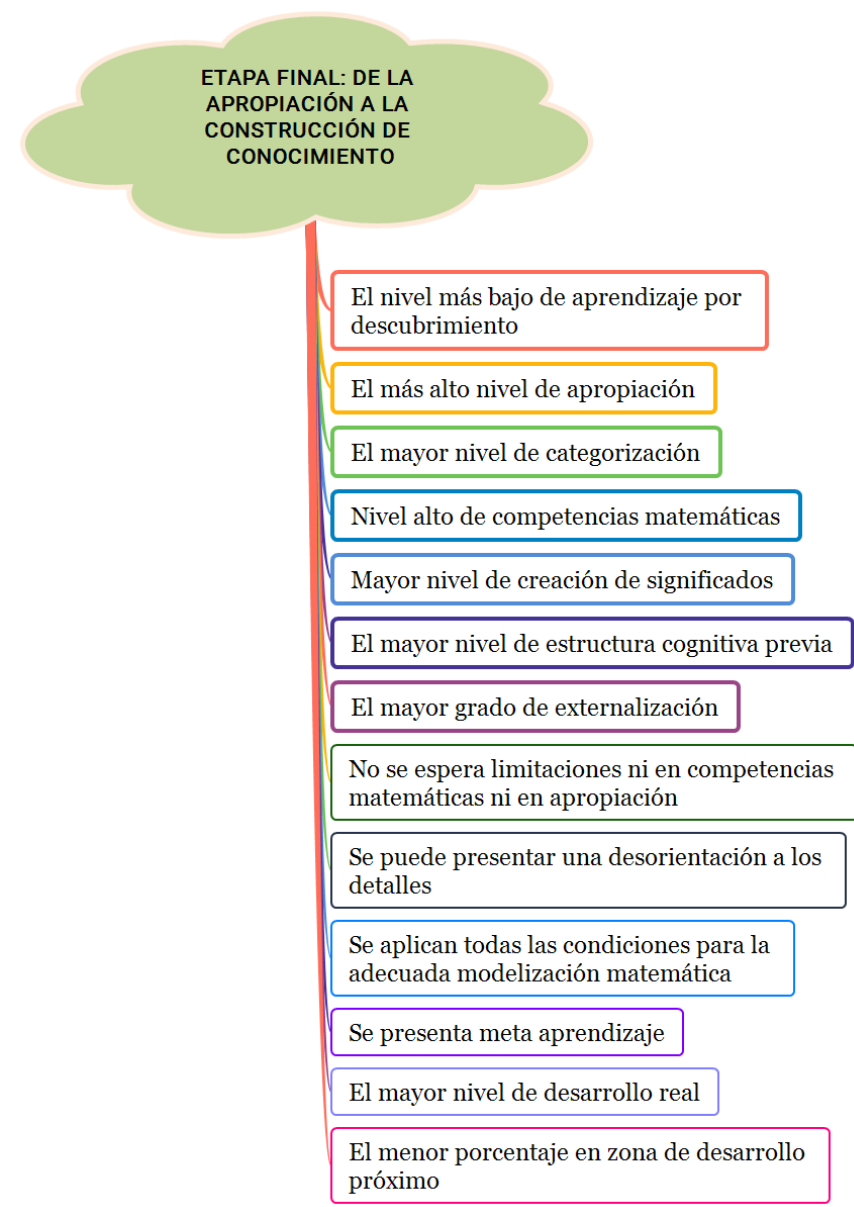

Fig. 6. Caracterización de la etapa final de la unidad didáctica [9] 
Es necesario aclarar que en esta etapa se presentó una particularidad, que no aparece en las anteriores: se trata de la desorientación al detalle. A pesar de que los estudiantes exponen de manera correcta, clara y completa el proceso que ejecutan, y que descifran y entienden el funcionamiento y las características de las herramientas financieras para la toma de decisiones, cuando deben plantear la fórmula, a pesar de contar con ella desde el comienzo del ejercicio, manifiestan dificultades para aplicarla, que no son de cálculo ni por falta de apropiación de conocimiento, sino por falta de atención a los detalles del ejercicio. Si bien ello les impide alcanzar el meta-aprendizaje, sí alcanzan a ubicarse en la zona de desarrollo real.

\section{Propuesta: Unidad didáctica: arquitectura de ideas}

Es a partir de los hallazgos que se propone la unidad didáctica, que consiste en un plan concreto de intervención en el aula, que abarca a la formación universitaria. Se caracteriza por hacer de la metodología de enseñanza-aprendizaje propuesta una herramienta de transformación, que procura al individuo la apropiación de conceptos y le facilita la construcción de conocimiento para aplicar en el mundo real.

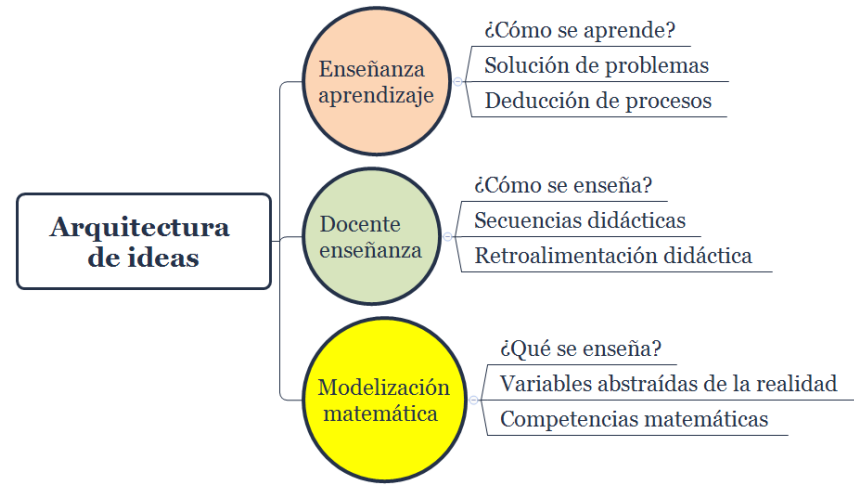

Fig. 7. Unidad didáctica, arquitectura de ideas. [9]

El modelo de construcción de conocimiento propuesto se ha denominado arquitectura de ideas. Su intención es convertir al estudiante, a través de la modelización matemática, en un sujeto activo en el proceso de enseñanzaaprendizaje. En términos generales, se abordan tres preguntas: ¿cómo se aprende?, ¿cómo se enseña? y ¿qué se enseña? No se acometerán explícitamente sino a través de la ampliación de teorías, como la enseñanza cooperativa y el aprendizaje significativo, y las percepciones que median el accionar del estudiante.

Para ello, se demarca la propuesta de aprendizaje, en cuanto acción fundadora del quehacer docente. En su libro Diferencia y repetición [10], Deleuze, citado por Urbina (2012) [11], explica con un ejemplo su concepto de aprendizaje:

(...) ¿Qué es aprender a nadar? Aprender a nadar implica una conjugación de ciertos puntos del cuerpo humano con los puntos singulares de la idea objetiva (el mar), para construir un campo problemático. Esa conjugación permite la toma de conciencia en la que los actos se acoplan a las percepciones del sujeto que aprende a nadar, favoreciendo la solución del problema (p. 121).

Acoplar la noción de aprendizaje de Deleuze [10] a la problemática de la investigación conlleva que el estudiante conjugue las experiencias previas en la identificación de las condiciones de su realidad, para equiparar las variables que afectan el comportamiento de su objeto de estudio. Este proceso construye el campo problemático y la toma de conciencia de estas variables, y la identificación de su comportamiento constituye las competencias matemáticas que le permiten generar un modelo matemático que representa las condiciones de la realidad, y le permiten aproximarse a una respuesta. En este ejercicio se produce una toma de conciencia entre la naturaleza y el espíritu, o, en el caso de la investigación, de la realidad y el sujeto, creándose una sensibilidad, «una especie de segunda potencia que capta lo que solo puede ser sentido» ([10], p. 252). Así, aprender implica la apropiación de conceptos, la transferencia de la internalización a la externalización, la deducción de procesos a través de pensamiento crítico, la resolución de problemas y la reflexión sobre los mismos, conjugando el paso de la zona de desarrollo próximo a la zona de desarrollo real.

En sintonía con el párrafo anterior, autores como Bruner [2] plantean que en el aprendizaje por descubrimiento el instructor debe motivar a los estudiantes para que sean ellos mismos los que descubran las relaciones entre conceptos, y construyan conocimientos. La reafirmación del papel activo del estudiante hace que se apropie del conocimiento, construya significados, establezca relaciones y desarrolle competencias de identificación de variables, que le permitan simplificar la realidad para modelarla y transformarla.

La construcción de conocimiento se logra a partir de la interacción del estudiante con el medio. Es un proceso en el cual el conocimiento es fruto de una reconstrucción continua, que, a su vez, es el resultado del intercambio del sujeto con el medio físico [1]. Por tanto, es fundamental la inclusión de la modelización matemática en la construcción de conocimiento, como un proceso de organización y representación de la realidad, en el desarrollo de competencias que permitan la clasificación de la información obtenida del medio. Según Vygotsky [3], «el aprendizaje organizado se convierte en desarrollo mental y pone en marcha una serie de procesos evolutivos que no pueden darse al margen del aprendizaje» ( $\mathrm{p}$. 12). Desde esta perspectiva, la inclusión de la modelización matemática facilita la elaboración de un aprendizaje organizado, permite el logro del desarrollo mental y supone la construcción de conocimiento como un proceso continuo de interacción.

Como la construcción de conocimiento es un proceso arduo para los estudiantes, esta intervención busca eliminar los elementos tensionantes de la experiencia formativa, propende por la cómoda y segura externalización de las ideas, y los invita a salir de la zona de confort. Es un instrumento que aspira a que los estudiantes se atrevan a arriesgarse y a emprender nuevas maneras de aprendizaje, en un juicio que les permita conocerse a sí mismos y monitorear sus propias 
acciones, sin el temor de un juicio externo y la preocupación por una nota. Siguiendo los planteamientos de Maaß [5], es un esfuerzo por transformar el aula en un espacio de metaaprendizaje, en el que se incentive la «motivación y la fuerza de voluntad» (p. 118), criterios que, para el autor, son fundamentales para el desarrollo de conocimientos estratégicos para resolver problemas y, de trasfondo, para el logro del meta-aprendizaje [5].

En este orden de ideas y dada la buena recepción entre los estudiantes de la metodología utilizada en la unidad didáctica, una propuesta resultante del proceso considera una secuencia didáctica que comprende los siguientes pasos:

- Introducción a la temática y relación de conceptos. Es un procedimiento inclusivo en el que el estudiante construye relaciones a partir de sus experiencias personales y deduce procedimientos para la solución de ejercicios matemáticos.

- Discusión de los conceptos entendidos por el estudiante. Consiste en la motivación hacia la externalización. Por medio de talleres, se pregunta sobre conceptos específicos o procedimientos, pero desde las propias palabras del estudiante. La nota se asigna por la justificación de los conceptos, la deducción de los procesos y la manera como el estudiante externaliza sus ideas, y no por la cercanía a la realidad del concepto y del proceso. Con estas actividades se desea eliminar la presión del error y motivar al estudiante a tomar riesgos para aprender, saliéndose de los esquemas dados por el profesor. La metodología se soporta en los planteamientos de Maaß [5], quien valiéndose de las concepciones de Sjuts (2003), indica que «el aula debe estar marcada por el discurso, el intercambio de percepciones individuales, la discusión sobre diferentes argumentos y la aclaración cognitiva, logrado a partir de una discusión en el aula o en tareas que analicen los errores» (p. 118).

- Apropiación y categorización de conceptos. A partir de la retroalimentación del docente y los demás compañeros de clase, se concibe, en términos comunes para todos los estudiantes, una metodología que busca que el estudiante tome conciencia de las diferencias entre los conceptos y procesos generados autónomamente y los brindados por el profesor, a fin de que esta conciencia permita el control del propio pensamiento y la concepción de metaaprendizaje ([5], p. 118) como factor para la construcción de conocimiento.

- Modelización matemática como recurso didáctico. Es un ejercicio en el que interactúan el docente, los estudiantes y los contenidos. Se caracteriza por la identificación y representación de la realidad bajo conceptos de simplificación, que posibilitan un proceso organizado de aprendizaje y la construcción de conocimiento.

- Evaluaciones en equipos de trabajo sobre la percepción de los estudiantes. La evaluación grupal facilita la externalización de conceptos y procesos. Al interactuar con los compañeros, los participantes deben concertar conceptos y concebir procedimientos comunes para elaborar sus respuestas, lo que implica mayor esfuerzo y mayor nivel de comprensión y apropiación de conocimientos. Y es que, de acuerdo con Maßß [5], «el trabajo en pequeños grupos y la discusión en grupos y de manera independiente soporta el desarrollo de competencias de modelización matemática, mostrando un impacto positivo, en el que se debe manejar el contexto, para motivar y no distraer» (p. 119).

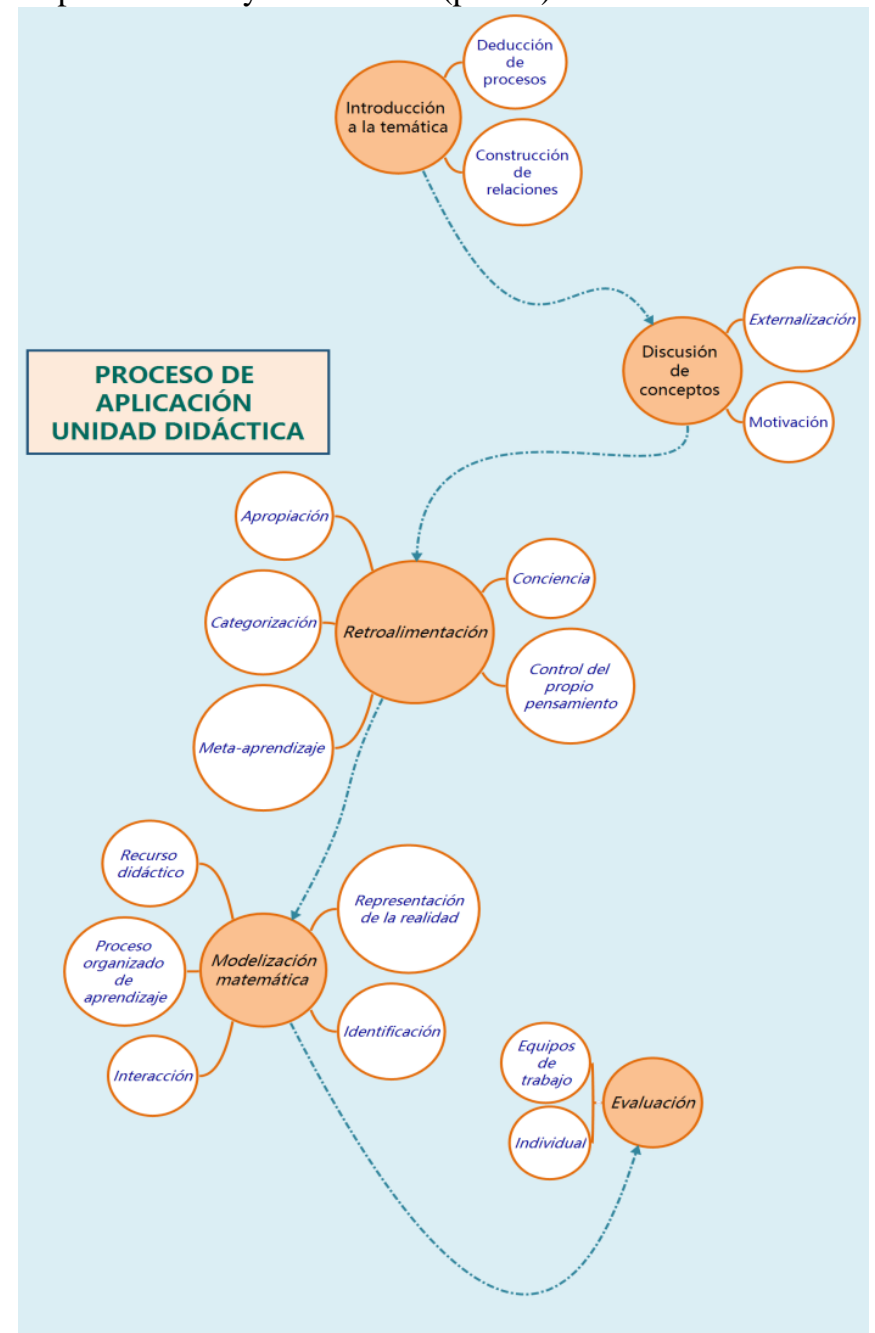

Fig. 8. Procedimiento en unidad didáctica. [9]

\section{CONCLUSIONES}

La investigación encuentra sus comienzos en el interés de interpretar si hay una verdadera incidencia de la modelización matemática sobre la construcción de conocimiento, entendiendo este término desde el cognitivismo y el constructivismo. A partir de estos enfoques se da inicio a un proceso investigativo, que se mueve entre algunos criterios cercanos y otros que se creen en olvido. Así, mediante la aplicación de una unidad didáctica, se busca identificar la cercanía de estas categorizaciones, y su aplicación y validez.

Desde el cognitivismo, se asumen como criterios cercanos la motivación, las expectativas y los conocimientos previos. En la teoría, estos criterios se asocian con el aprendizaje 
significativo [12], porque constituyen elementos cruciales para la construcción de conocimiento; en la práctica, aportan evidencia sobre el impacto que la unidad didáctica tuvo en los estudiantes del curso de ingeniería económica de la Universidad Tecnológica de Pereira.

En el curso de la actividad, los estudiantes logran la apropiación, la categorización de la información, la trascendencia hacia zonas de desarrollo y el meta-aprendizaje, al transformar el aula de clase en un espacio de interacción. El resultado es que el estudiante logra asumir un rol activo en el proceso de aprendizaje, mientras que el docente se convierte en un agente facilitador, que incentiva el trabajo en equipo como estrategia para suprimir la presión, externalizar los significados y construir concertadamente conceptos comunes desde roles de conflicto y apoyo, que son elementos que soportan el aprendizaje cooperativo [13]. Así las cosas, la propuesta encuentra amplia aceptación entre los estudiantes.

La propuesta de direccionar la construcción de conocimiento desde procesos de deducción matemática, en los que el estudiante desarrolla un pensamiento lógico y relaciona los saberes aprendidos con los conceptos por saber [12], se conecta con los planteamientos del constructivismo basados en procesos internos del sujeto y la percepción del entorno.

Los criterios en olvido que estas temáticas reconocen parten de la enseñanza, el aprendizaje, el reconocimiento del individuo y el conocimiento mismo. Es una identificación que, en la unidad didáctica, proviene del momento en que los estudiantes asumen las clases como un proceso de transferencia de información, antes que como una oportunidad para construir conocimiento, apropiar conceptos y juzgar su propio aprendizaje. Es un enfoque que propicia una clase magistral, en la que los conceptos no se construyen sino que se trasmiten.

La metodología propuesta por esta investigación es de gran aceptación. A decir de los estudiantes, la metodología facilita el desarrollo de nuevos conocimientos, la formación de posturas críticas frente a la realidad en la que deviene su experiencia, e, incluso, la emisión de juicios sobre su propio proceso de aprendizaje; asimismo, indican que es una estrategia más aplicada porque aprovecha sus propios conceptos y deducciones, y no tienen que esperar a que el profesor se los revele.

La modelización matemática se instituye como una metodología que forma en competencias cruciales para el entendimiento y la simplificación de la realidad [5], situación que se fortalece significativamente en el estudiante cuando comprende que los aprendizajes adquiridos en su experiencia formativa tienen aplicación práctica en su diario vivir [6]. Cuando el estudiante encuentra que su conocimiento es relevante para la sociedad, se estimula a seguir aprendiendo y a profundizar sobre lo aprendido [14], de lo que se sigue que la modelización matemática también representa un factor motivacional en la construcción de conocimiento.

Como colofón de la investigación: La enseñanza en el aula de clases universitaria es una construcción pedagógica que tiene en cuenta al individuo, su formación y su interacción con el medio, se pudo evidenciar cómo los procesos de enseñanza-aprendizaje son enriquecedores, a partir de pautas como: el aprendizaje cooperativo, la construcción de conceptos comunes en roles de conflicto con participación activa, los procesos desde la realidad, las estrategias que conllevan suprimir la presión y el significado en el aprendizaje. Es una transición que implica pensar, interactuar y replantear al otro como un ser, como un personaje activo en el proceso formativo. Solo a partir de allí podrá alcanzarse la genuina construcción pedagógica y la verdadera construcción de conocimiento.

\section{RECOMENDACIONES}

Si bien este estudio intenta comprender la interacción de la modelización matemática dentro del proceso de construcción de conocimiento, existen otros factores emergentes por incorporar dentro del análisis del marco metodológico. Se evidencian otras alusiones por considerar en el proceso de mejora de la enseñanza-aprendizaje que, con suerte, conllevan la formulación de otras preguntas de investigación, que pueden ofrecer una mayor claridad frente a la construcción de conocimiento, construcción entendida desde el cognitivismo y constructivismo. Son alusiones que parten desde el aprendizaje significativo y los procesos internos de aprendizaje del estudiante.

Al tomar los resultados de esta indagación, un análisis futuro puede procurar evidenciar el efecto del grado de desarrollo de los conocimientos previos en el proceso de construcción de conocimiento. Con ese fin, sería pertinente examinar si se presentan baches significativos en el proceso de construcción de conocimiento en estudiantes que pueden presentar falencias de apropiación, frente a aquellos que presentan un mayor grado de apropiación de conocimientos previos. Esa identificación haría posible la formulación de estrategias de compensación en el aula conducentes a la nivelación de aquellos estudiantes rezagados.

Si bien es cierto que este estudio asumió el examen de un segmento del proceso de enseñanza-aprendizaje en el ámbito de la docencia universitaria, valdría la pena examinar el mismo proceso en el ámbito de la educación básica y media. De hacerlo, sería posible evidenciar el efecto de la modelización matemática en la construcción de conocimiento en una muestra de estudiantes que probablemente ingresarán a la universidad, logrando un panorama más amplio y global del sistema educativo, que potencie la construcción de conocimiento a temprana edad.

\section{REFERENCIAS}

[1] S. Parrat-Dayan, "Esencia y trascendencia de la obra de Jean Piaget (1896-1980)", Persona, no. 15, pp. 213-224, 2012 ISSN: 1560-6139. https://revistas.ulima.edu.pe/index.php/Persona. DOI: 10.26439/persona

[2] M. Guilar, "Las ideas de Bruner de la revolución cognitiva a la revolución cultural", Educere, vol. 13, no. 44, pp. 235-241, 2009 ISSN: 1316-4910. http://www.human.ula.ve/adocente/educere/

[3] L. Vygotsky, El desarrollo de los procesos psicológicos superiores. Barcelona, España: Crítica, 1978.

[4] P. Ertmer, y T. Newby, "Conductismo, cognitivismo y constructivismo: una comparación de los aspectos críticos desde la perspectiva del diseño de introducción", Performance Improvement Quarterly, vol. 6, no. 4, pp. 50-72, 1993. DOI: 10.1111/j.1937-8327.1993.tb00605.x. 
[5] K. Maaß, "What are modelling competencies?", International journal on mathematics education, ZDM. vol. 38, no. 2, pp. 113-142, 2006. ISSN: 1863-9704. DOI: http://doi.org/10.1007/BF02655885.

[6] G. Kaiser, y B. Schwarz, "Mathematical modelling as bridge between School and University", International journal on mathematics education, ZDM, vol. 38, no. 2, pp. 196-208, 2006. ISSN: 1863-9704. DOI: 10.1007/BF02655885.

[7] M. Rodríguez, "La teoría del aprendizaje significativo", en Concept maps: Theory, methodology, Technology, Vol. 1. Pamplona, España: Dirección de publicaciones de la Universidad Pública de Navarra, 2004, pp. 535544. ISBN: 84-9769-064-8.

[8] R. Krueger, Focus group: a practical guide for applied research. Thousand Oask, California: Sage, 1994.

[9] L. Arias, "Construcción de conocimiento en alumnos universitarios a partir de la modelización matemática", tesis doctoral, Univ. Tecnológica de Pereira, Pereira, 2019.

[10] G. Deleuze, Diferencia y repetición. Buenos Aires: Amorrortu, 2002.

[11] J. Urbina, "La pasión de aprender. El punto de vista de los estudiantes universitarios", tesis doctoral, Univ. de Manizales, 2012, [En línea]. Disponible en: https://studylib.es/doc/6304544/la-pasi\%C3\%B3n-deaprender.-el-punto-de-vista-de-los

[12] F. Romero, "Aprendizaje significativo y constructivismo", Temas para la educación, no. 3, 2009. ISSN: 1989-4023 [En línea]. https://www.feandalucia.ccoo.es/indicei.aspx? $\mathrm{p}=62 \& \mathrm{~d}=176 \& \mathrm{~s}=5$

[13] A. Brown y A. Palincsar, "Guided, cooperative learning and individual knowledge acquisition", Institute of Education Sciences, Tech. Rep.; No. 372, 1986 [online]. Available: https://eric.ed.gov/?id=ED270738. [Accessed: Jun 29,-2018]

[14] M. Triguero, "El uso de la modelación en la enseñanza de las matemáticas", Innovación educativa, vol. 9, no. 46, pp. 75-87, 2009. ISSN: 1665-2673. www.innovacion.ipn.mx. [Accedido: 15-Sep-2017]

[15] J. Bruner, El proceso de la educación. México: UTEHA, 1963.

[16] J. Bruner, J. Goodnow, y G. Austin, El proceso mental en el aprendizaje. Madrid: Narcea, 1978.

[17] M. Ledesma, Análisis de la teoría de Vygotsky para la reconstrucción de la inteligencia social. Universidad Católica de Cuenca. 2014. ISBN: 9789942-9860-9-2. www.ucacue.edu.ec

[18] L. Vygotsky, "Consciousness as a problem in the psychology of behavior", Journal of Russian and East European Psychology, vol. 17 no.4, pp. 3-35, 1979. DOI: 10.2753/RPO1061-040517043

[19] S. Merriam, Qualitative research and case study applications in education. San Francisco: Jossey-Bass, 1998.

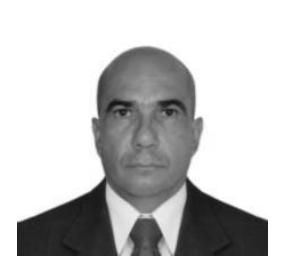

Leonel Arias-Montoya, Ingeniero Industrial de la Universidad Tecnológica de Pereira (1991), Especialista en Administración del Desarrollo Humano (2002), Magíster en Administración Económica y Financiera (2004), Candidato a Doctor en Didáctica de la Universidad Tecnológica de Pereira. Es Profesor Titular del programa de Ingeniería Industrial de la Universidad Tecnológica de Pereira desde el año 2006. Ha sido director de Maestría en Administración Económica y Financiera, desde el 2008 hasta el 2013 y de la Especialización en Gerencia de Proyectos desde enero de 2019.

ORCID: https://orcid.org/0000-0002-1485-4651

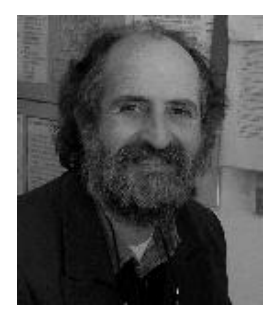

Jordi Deulofeu-Piquet, licenciado en Matemáticas (U. de Barcelona, 1976) y Doctor en Didáctica de las Matemáticas (U. Autònomade Barcelona, 1993). Profesor del Departamento de Didáctica de la Matemática de la UAB desde 1978. Director del Máster de Secundaria de
Matemáticas. Investigación en: Competencia matemática, Resolución de problemas y Estudio de la práctica del profesor de matemáticas.

ORDID: https://orcid.org/0000-0002-5834-0863 